Table 1. Clinical and laboratory characteristics of AS activity at baseline and week $52, n=30$

\begin{tabular}{lccc}
\hline & Baseline & Week 52 & $\mathrm{p}$ \\
\hline Pain, Me [Q25;Q75] & $6.0[7.0 ; 9.0]$ & $3.4[1.0 ; 5.0]$ & $<0.000$ \\
BASDAI, Me [Q25;Q75] & $6.6[4.4 ; 8.85]$ & $3.2[2.1 ; 7.6]$ & $<0.000$ \\
ASDAS, Me [Q25;Q75] & $3.8[3.5 ; 4.4]$ & $2.1[1.26 ; 4.26]$ & $<0.000$ \\
C-RP, mg/l, M $\pm S D$ & $12.3 \pm 3.9$ & $4.3 \pm 1.7$ & $<0.000$ \\
TNF $\alpha, \mathrm{pg} / \mathrm{ml}, \mathrm{M} \pm$ SD & $17.8 \pm 7.6$ & $7.3 \pm 3.2$ & $<0.000$ \\
IL-17A, pg/ml, M \pm SD & $28.4 \pm 14.4$ & $32.1 \pm 12.2$ & 0.29 \\
\hline
\end{tabular}

AS achieved ASAS partial remission. IL-17A was lower in patients who achieved remission compared to patients who did not achieve remission (figure).

Conclusions: Serum concentration of IL-17A remains stable in patients treated with anti-TNF $\alpha$ during the year. The baseline and final concentrations of serum IL-17A are higher in patients with AS who do not achieved ASAS partial remission compared to those who achieved the remission.

Disclosure of Interest: None declared

DOI: 10.1136/annrheumdis-2017-eular.5305

\section{THU0371 THERAPY MODIFICATIONS IN PATIENTS WITH ANKYLOSING SPONDYLITIS TREATED WITH A BIOLOGIC IN THE UNITED STATES - DESCRIPTIVE ANALYSES FROM AN ADMINISTRATIVE CLAIMS DATABASE}

J.A. Walsh ${ }^{1}$, O. Adejoro ${ }^{2}$, B. Chastek ${ }^{2}$, G. Chun ${ }^{3}$, Y. Park ${ }^{3} .{ }^{1}$ University of Utah School of Medicine, Salt Lake City, UT; ${ }^{2}$ Optum, Eden Prairie, MN; ${ }^{3}$ Novartis Pharmaceuticals Corporation, East Hanover, NJ, United States

Background: For patients with active ankylosing spondylitis (AS), biologic therapy has been shown to be an effective treatment option. Physicians treating patients with biologic therapy may modify the treatment by adding non-biologic medications and/or escalating the dose of the biologic; however, limited data exist on how these therapy modifications are used in patients with AS receiving biologic treatment in real-world settings.

Objectives: To describe therapy modifications (adding non-biologic medications or dose escalation of the biologic therapy) in patients with active AS who newly initiated treatment with biologic therapy in the United States.

Methods: This study used US administrative pharmacy and medical claims data from the Optum Research Database. Adult patients with AS who newly initiated (no evidence of use in the 12 months prior) a biologic between January 1, 2013 and January 31,2015 , and were continuously enrolled in a commercial or Medicare Advantage health plan 12 months before (baseline period) and 15 months following the index date, defined as the date of first pharmacy fill or medical infusion, were included. To reduce confounding by patients with an early switch/discontinuation, therapy modifications were identified only in those who persisted on the index biologic for $>90$ days. Therapy modifications identified included initiation of add-on medications (disease-modifying antirheumatic drugs, nonsteroidal antiinflammatory drugs [NSAIDs], opioids, corticosteroids, antidepressants, anxiolytics, sleeping aids and topical analgesics) after the first 90 days of persistence, and dose escalation of the index biologic. Dose escalation was defined as a patient receiving a dose $>10 \%$ above the reference dose from the product label for $\geq 90$ days. Results: Of the 333 patients with AS included who persisted on their index biologic for $>90$ days, $88.3 \%$ initiated a subcutaneous tumor necrosis factor inhibitor (TNFi-SC; adalimumab, certolizumab pegol, etanercept or golimumab) as their index biologic and $11.7 \%$ initiated an intravenous TNFi (TNFi-IV; infliximab). During the 12-month baseline period, patients had a mean (standard deviation) number of claims of 3.9 (6.6) for opioids, followed by 2.5 (3.1) for NSAIDs, 1.9 (2.8) for corticosteroids and 1.8 (3.7) for antidepressants. Overall, $44.7 \%$ of patients received $\geq 1$ additional medication during the period from 90 days after the index date to the end of persistence with the index biologic or 12-month post-index period. The most commonly added medications were corticosteroids $(16.8 \%)$, opioids $(12.9 \%)$, NSAIDs $(10.2 \%)$ and antidepressants $(7.2 \%)$ (Table 1). Overall, $7.2 \%$ of patients had a dose escalation of the index biologic (38.5\% for TNFi-IV and $2.7 \%$ for TNFi-SC) in the immediate 12-month post-index period.

Table 1. Add-on medications initiated from 90 days after the index date to the end of persistence or 12 months among patients with AS

\begin{tabular}{|c|c|c|c|}
\hline Additional Medication, $\mathrm{n}(\%)$ & $\begin{array}{c}\text { Total } \\
(\mathrm{N}=333)\end{array}$ & $\begin{array}{c}\text { TNFi-SC } \\
(n=294)\end{array}$ & $\begin{array}{l}\text { TNFi-IV } \\
(n=39)\end{array}$ \\
\hline Any medication & $149(44.7)$ & $129(43.9)$ & $20(51.3)$ \\
\hline Corticosteroid & $56(16.8)$ & $46(15.6)$ & $10(25.6)$ \\
\hline Opioid & $43(12.9)$ & $39(15.5)$ & $4(10.3)$ \\
\hline NSAID & $34(10.2)$ & $30(10.2)$ & $4(10.3)$ \\
\hline Antidepressant & $24(7.2)$ & $22(7.5)$ & $2(5.1)$ \\
\hline Anxiolytic & $23(6.9)$ & $18(6.1)$ & $5(12.8)$ \\
\hline cSDMARD & $15(4.5)$ & $13(4.4)$ & $2(5.1)$ \\
\hline Topical analgesic & $13(3.9)$ & $9(3.1)$ & $4(10.3)$ \\
\hline Sleeping aid & $9(2.7)$ & $8(2.7)$ & $1(2.6)$ \\
\hline tsDMARD & $0(0.0)$ & $0(0.0)$ & $0(0.0)$ \\
\hline
\end{tabular}

AS, ankylosing spondylitis; csDMARD, conventional synthetic disease-modifying antirheumatic drug; IV, intravenous; SC, subcutaneous; TNFi, tumor necrosis factor inhibitor; tsDMARD, targeted synthetic disease-modifying antirheumatic drug. *TNFi-SC includes adalimumab, certolizumab, etanercept and golimumab. t TNFi-IV includes infliximab.
Conclusions: In this descriptive, administrative claims-based study from the US, approximately $45 \%$ of patients with AS initiated an add-on medication while receiving biologic therapy. Further research is needed to better understand optimal therapy strategies for patients with AS.

Acknowledgements: This study was sponsored by Novartis Pharmaceuticals Corporation, East Hanover, NJ.

Disclosure of Interest: J. Walsh Consultant for: Novartis, O. Adejoro Employee of: Optum, B. Chastek Employee of: Optum, G. Chun Employee of: Novartis, Y. Park Employee of: Novartis

DOI: 10.1136/annrheumdis-2017-eular.3035

\section{THU0372 EFFECT OF TNFI VERSUS NSAID ON SPINAL RADIOGRAPHIC PROGRESSION OVER 4 YEARS IN EARLY ANKYLOSING SPONDYLITIS: RESULTS FROM TWO OBSERVATIONAL COHORTS IN SOUTH KOREA}

J.W. Park ${ }^{1}$, M.J. Kim ${ }^{1}$, J.K. Park ${ }^{1}$, Y.J. Lee ${ }^{2}$, E.B. Lee ${ }^{1}$, Y.W. Song ${ }^{1}$, E.Y. Lee ${ }^{1}$ ${ }^{1}$ Division of Rheumatology, Department of Intermal Medicine, Seoul National University Hospital, Seoul; ${ }^{2}$ Division of Rheumatology, Department of Intermal Medicine, Seoul National University Bundang Hospital, Gyeonggi-do, Korea, Republic Of

Background: It is uncertain whether early suppression of inflammation by tumor necrosis factor inhibitor (TNFi) leads to a decreased radiographic progression in axial spondyloarthritis (axSpA).

Objectives: To compare the radiographic progression over 4 years in patients with early ankylosing spondylitis (AS) using TNFi versus nonsteroidal antiinflammatory drug (NSAID)

Methods: A total of 215 patients with early AS (symptom duration $<10$ years) were included based on the availability of radiographs at baseline and 2and/or 4-years of follow up. Among them, 135 patients with TNFi were from SNUH-biologics cohort (TNFi group) and other 80 patients with NSAID were from control cohort in Seoul National University Bundang Hospital (NSAID group). Radiographic progression was assessed by two blinded readers using modified Stokes AS Spinal Score (mSASSS). Linear mixed model was applied to compare the radiographic progression between the two groups after adjustment for clinical factors. We also performed a sensitivity analysis after the propensity score matching in which age, smoking status, baseline CRP and baseline mSASSS were included as covariates.

Results: Patients in the TNFi group showed higher baseline BASDAI (6.7 vs. 3.1 ) and CRP (2.2 vs. $1.1 \mathrm{mg} / \mathrm{dL}$ ) as compared with those in the NSAID group. There were no differences between the two groups regarding age, gender, HLAB27, smoking status and baseline radiographic damage. Overall, radiographic progression rate $(95 \% \mathrm{Cl})$ during the observation was $0.72(0.57-0.87)$ unit/year. TNFi group showed significantly slower progression than NSAID group ( $\beta=-0.33$ unit/year, $p=0.042$ ). This result was consistent after adjusting for age, smoking status, baseline CRP and presence of baseline syndesmophytes $(\beta=-0.50$ unit/year, $p=0.001$ ) (Table). In the subgroup analysis of patients without baseline syndesmophytes, TNFi group showed no radiographic progression over time whereas NSAID group did not (0.03 [-0.22-0.27] vs. 0.45 [0.20-0.71] unit/year). These results were not changed when the same analysis was performed in the post-matched population (78 TNFi group vs. 78 NSAID group).

Table 1. Radiographic progression over time in early AS patients using NSAID vs. TNFi

\begin{tabular}{lcclccc}
\hline & \multicolumn{2}{c}{ Univariable analysis } & & \multicolumn{2}{c}{ Multivariable analysis $^{\mathrm{b}}$} \\
\cline { 2 - 3 } \cline { 5 - 6 } & $\begin{array}{c}\text { Regression } \\
\text { coefficient }(95 \% \mathrm{Cl})^{\mathrm{a}}\end{array}$ & $p$ value & & $\begin{array}{c}\text { Regression } \\
\text { coefficient }(95 \% \mathrm{Cl})\end{array}$ & $p$ value \\
\hline Age $>40$ & $0.82(0.48-1.16)$ & $<0.001$ & & $0.27(-0.07-0.61)$ & 0.124 \\
Ever-smoker & $0.31(-0.01-0.62)$ & 0.056 & & $0.08(-0.20-0.37)$ & 0.568 \\
Baseline CRP (mg/dL) & $0.12(0.05-0.18)$ & 0.001 & & $0.10(0.04-0.16)$ & 0.002 \\
Baseline syndesmophytes & $1.30(1.00-1.62)$ & $<0.001$ & & $1.14(0.80-1.48)$ & $<0.001$ \\
Group & $-0.33(-0.65-0.01)$ & 0.042 & & $-0.50(-0.79-0.22)$ & 0.001 \\
NSAID group & $0.93(0.68-1.18)$ & $<0.001$ & & $0.45(0.18-0.71)$ & 0.001 \\
TNFi group & $0.60(0.40-0.79)$ & $<0.001$ & & $-0.06(-0.31-0.19)$ & 0.648 \\
\hline
\end{tabular}

${ }^{a}$ Regression coefficient indicates the progression of mSASSS over one year. ${ }^{b}$ Gender, HLAB27, baseline BASDAI and time-averaged NSAID index were not included because they did not show a significant $(p<0.1)$ interaction with time in the univariable analysis.

Conclusions: In patients with early AS, TNFi led to a decreased radiographic progression as compared with NSAID treatment. This result suggests that early and durable suppression of inflammation using TNFi can have beneficial effect on the radiographic outcome of AS.

Disclosure of Interest: None declared

DOI: 10.1136/annrheumdis-2017-eular.3321 